\title{
Generalized Wigner-Yanase Skew Information And Generalized Fisher Information
}

\author{
Kenjiro YANAGI ${ }^{\ddagger}$, Shigeru FURUICHI ${ }^{\dagger}$ and Ken KURIYAMA ${ }^{\ddagger}$ \\ $\ddagger$ Applied Mathematical Science, \\ Graduate School of Science and \\ Engineering, Yamaguchi University, \\ 2-16-1, Tokiwadai, Ube, 755-8611, Japan \\ E-mail: yanagi@yamaguchi-u.ac.jp \\ E-mail: kuriyama@yamaguchi-u.ac.jp \\ $\dagger$ Department of Computer Science and \\ System Analysis, College of Humanities \\ and Sciences, Nihon University, \\ 3-25-40, Sakurajyousui, Setagaya-ku, \\ Tokyo, 156-8550, Japan \\ E-mail: furuichi@cssa.chs.nihon-u.ac.jp
}

\begin{abstract}
We introduce a generalized Wigner-Yanase skew information and then derive the trace inequality related to the uncertainty relation. This inequality is a non-trivial generalization of the uncertainty relation derived by S.Luo for the quantum uncertainty quantity excluding the classical mixture. And we introduce a generalized Fisher information and then derive a generalized Cramér-Rao inequality. We also give an example for our generalized Fisher information and then derive the uncertainty relation for two observables.
\end{abstract}

\section{INTRODUCTION}

As a degree for non-commutativity between a quantum state $\rho$ and an observable $H$, Wigner-Yanase skew information

$$
I_{\rho}(H) \equiv \frac{1}{2} \operatorname{Tr}\left[\left(i\left[\rho^{1 / 2}, H\right]\right)^{2}\right]
$$

was defined in [10]. Here we denote the commutator by $[X, Y]=X Y-Y X$. This quantity was generalized by Dyson

$$
I_{\rho, \alpha}(H)=\frac{1}{2} \operatorname{Tr}\left[\left(i\left[\rho^{\alpha}, H\right]\right)\left(i\left[\rho^{1-\alpha}, H\right]\right)\right]
$$

which is known as the Wigner-Yanase-Dyson skew information. It is famous that the convexity of $I \rho, \alpha(H)$ with respect to $\rho$ was successfully proven by E.Lieb in [7]. From the physical point of view, an observable $H$ is generally considered to be an unbounded operator, however in the present paper, unless otherwise stated, we consider $H \in B(\mathcal{H})$, where $B(\mathcal{H})$ represents the set of all bounded linear operators on the Hilbert space

This work was partially supported by Grant-in-Aids for Scientific Research (C), 20540175, 20540176 and (B), 18300003.
$\mathcal{H}$, as a mathematical interest. We also denote the set of all self-adjoint operators (observables) by $\mathcal{L}_{h}(\mathcal{H})$ and the set of all density operators (quantum states) by $\mathcal{S}(\mathcal{H})$ on the Hilbert space $\mathcal{H}$. The relation between the Wigner-Yanase skew information and the uncertainty relation was studied in [9]. Moreover the relation between the Wigner-Yanase-Dyson skew information and the uncertainty relation was studied in $[6,11]$. In our previous paper [11], we defined a generalized skew information and then derived a kind of an uncertainty relation. In the section 2 , we introduce a new generalized Wigner-Yanase-Dyson skew information. On a generalization of the original Wigner-Yanase-Dyson skew information, our generalization is different from the Wigner-Yanase-Dyson skew information and a generalized skew information defined in our previous paper [11].

On the other hand, we have some definitions for the Fisher information in quantum mechanical system. In the section 3 , we consider the standard definition and its one-parameter extended one. For a parameterized density operator $\rho_{\theta} \in \mathcal{S}_{\theta}(\mathcal{H})$, we define the Fisher information by

$$
I\left(\rho_{\theta}, L_{\theta}\right) \equiv \operatorname{Tr}\left[\rho_{\theta} L_{\theta} L_{\theta}^{*}\right],
$$

where the logarithmic derivative $L_{\theta}$ is defined by

$$
\frac{\partial \rho_{\theta}}{\partial \theta} \equiv \frac{1}{2}\left(\rho_{\theta} L_{\theta}+L_{\theta}^{*} \rho_{\theta}\right)
$$

and $\mathcal{S}_{\theta}(\mathcal{H})$ represents the set of all quantum states with one-parameter $\theta \in \mathbb{R}$. In the section 3 of the present paper, we define a one-parameter extended Fisher information and study some trace inequalities between this quantity and the variance (a generalized CramérRao type inequality). See the literatures $[2,3]$ on recent advances of the skew information, the Fisher information and the uncertainty relation. 


\section{TRACE INEQUALITIES ON A GENER- ALIZED WIGNER-YANASE SKEW INFOR- MATION}

We review the relation between the Wigner-Yanase skew information and the uncertainty relation. In quantum mechanical system, the expectation value of an observable $H$ in a quantum state $\rho$ is expressed by $\operatorname{Tr}[\rho H]$. It is natural that the variance for a quantum state $\rho$ and an observable $H$ is defined by $V_{\rho}(H) \equiv \operatorname{Tr}\left[\rho(H-\operatorname{Tr}[\rho H] I)^{2}\right]=\operatorname{Tr}\left[\rho H^{2}\right]-\operatorname{Tr}[\rho H]^{2}$. It is famous that we have the Heisenberg's uncertainty relation:

$$
V_{\rho}(A) V_{\rho}(B) \geq \frac{1}{4}|\operatorname{Tr}[\rho[A, B]]|^{2}
$$

for a quantum state $\rho$ and two observables $A$ and $B$. The further strong result was given by Schrödinger

$$
V_{\rho}(A) V_{\rho}(B)-\left|\operatorname{Cov}_{\rho}(A, B)\right|^{2} \geq \frac{1}{4}|\operatorname{Tr}[\rho[A, B]]|^{2},
$$

where the covariance is defined by $\operatorname{Cov}_{\rho}(A, B) \equiv$ $\operatorname{Tr}[\rho(A-\operatorname{Tr}[\rho A] I)(B-\operatorname{Tr}[\rho B] I)]$. However, the uncertainty relation for the skew information failed. (See $[9,6,11]$.

$$
I_{\rho}(A) I_{\rho}(B) \geq \frac{1}{4}|\operatorname{Tr}[\rho[A, B]]|^{2} .
$$

Recently S.Luo introduced the quantity $U_{\rho}(H)$ representing a quantum uncertainty excluding the classical mixture:

$$
U_{\rho}(H) \equiv \sqrt{V_{\rho}(H)^{2}-\left(V_{\rho}(H)-I_{\rho}(H)\right)^{2}} .
$$

Note that we have the relation among quantities as

$$
0 \leq I_{\rho}(H) \leq U_{\rho}(H) \leq V_{\rho}(H)
$$

For a quantum state $\rho$ and observables $X, Y$, he derived the following uncertainty relation in [8]:

$$
U_{\rho}(X) U_{\rho}(Y) \geq \frac{1}{4}|\operatorname{Tr}[\rho[X, Y]]|^{2} .
$$

The inequality (3) is a refinement of the inequality (1) in the sense of (2). In this section, we study two types of one-parameter extended inequalities for the inequality (3).

Definition 2.1 For $0 \leq \alpha \leq 1$, a quantum state $\rho$ and an observable $H$, we define the Wigner-Yanase-Dyson skew information

$$
I_{\rho, \alpha}(H) \equiv \frac{1}{2} \operatorname{Tr}\left[\left(i\left[\rho^{\alpha}, H_{0}\right]\right)\left(i\left[\rho^{1-\alpha}, H_{0}\right]\right)\right]
$$

and we also define

$$
J_{\rho, \alpha}(H) \equiv \frac{1}{2} \operatorname{Tr}\left[\left\{\rho^{\alpha}, H_{0}\right\}\left\{\rho^{1-\alpha}, H_{0}\right\}\right],
$$

where $H_{0} \equiv H-\operatorname{Tr}[\rho H] I$ and we denote the anticommutator by $\{X, Y\}=X Y-Y X$.

Note that we have

$$
\begin{aligned}
& \frac{1}{2} \operatorname{Tr}\left[\left(i\left[\rho^{\alpha}, H_{0}\right]\right)\left(i\left[\rho^{1-\alpha}, H_{0}\right]\right)\right] \\
= & \frac{1}{2} \operatorname{Tr}\left[\left(i\left[\rho^{\alpha}, H\right]\right)\left(i\left[\rho^{1-\alpha}, H\right]\right)\right],
\end{aligned}
$$

but we have

$$
\frac{1}{2} \operatorname{Tr}\left[\left\{\rho^{\alpha}, H_{0}\right\}\left\{\rho^{1-\alpha}, H_{0}\right\}\right] \neq \frac{1}{2} \operatorname{Tr}\left[\left\{\rho^{\alpha}, H\right\}\left\{\rho^{1-\alpha}, H\right\}\right] .
$$

Then we have the following inequalities:

$$
I_{\rho, \alpha}(H) \leq I_{\rho}(H) \leq J_{\rho}(H) \leq J_{\rho, \alpha}(H),
$$

since we have $\operatorname{Tr}\left[\rho^{1 / 2} H \rho^{1 / 2} H\right] \leq \operatorname{Tr}\left[\rho^{\alpha} H \rho^{1-\alpha} H\right]$. If we define

$$
U_{\rho, \alpha}(H) \equiv \sqrt{V_{\rho}(H)^{2}-\left(V_{\rho}(H)-I_{\rho, \alpha}(H)\right)^{2}},
$$

as a direct generalization of Eq.(1), then we have

$$
0 \leq I_{\rho, \alpha}(H) \leq U_{\rho, \alpha}(H) \leq U_{\rho}(H)
$$

due to the first inequality of (9). We also have

$$
U_{\rho, \alpha}(H)=\sqrt{I_{\rho, \alpha}(H) J_{\rho, \alpha}(H)} .
$$

In this paper, we introduce a generalized WignerYanase skew information which is a generalized Wigner-Yanase skew information by

$$
K_{\rho, \alpha}(H) \equiv \frac{1}{2} \operatorname{Tr}\left[\left(i\left[\frac{\rho^{\alpha}+\rho^{1-\alpha}}{2}, H_{0}\right]\right)^{2}\right]
$$

and we also define

$$
L_{\rho, \alpha}(H) \equiv \frac{1}{2} \operatorname{Tr}\left[\left(\left\{\frac{\rho^{\alpha}+\rho^{1-\alpha}}{2}, H_{0}\right\}\right)^{2}\right] .
$$

Throughout this section, we put $X_{0} \equiv X-\operatorname{Tr}[\rho X] I$ and $Y_{0} \equiv Y-\operatorname{Tr}[\rho Y] I$. Then we show the following trace inequality.

Theorem 2.2 For a quantum state $\rho$ and observable $X, Y$ and $\alpha \in[0,1]$, we have

$$
W_{\rho, \alpha}(X) W_{\rho, \alpha}(Y) \geq \frac{1}{4}\left|\operatorname{Tr}\left[\left(\frac{\rho^{\alpha}+\rho^{1-\alpha}}{2}\right)^{2}[X, Y]\right]\right|^{2}
$$

where

$$
W_{\rho, \alpha}(X) \equiv \sqrt{K_{\rho, \alpha}(X) L_{\rho, \alpha}(X)}
$$


Remark 2.3 Theorem 2.2 is not trivial by the following two reasons.

(1) There is no relation between

$$
\left|\operatorname{Tr}\left[\left(\frac{\rho^{\alpha}+\rho^{1-\alpha}}{2}\right)^{2}[X, Y]\right]\right|^{2}
$$

and $|\operatorname{Tr}[\rho[X, Y]]|^{2}$.

(2) Though $U_{\rho, \alpha}(H) \leq U_{\rho}(H)$ and $U_{\rho, \alpha}(H) \leq$ $\tilde{U}_{\rho, \alpha}(H)$ hold, there is no relation between $U_{\rho}(H)$ and $\tilde{U}_{\rho, \alpha}(H)$.

Proof of Theorem 2.2. We put

$$
A_{\alpha}(H) \equiv i\left[\rho^{\alpha}, H_{0}\right], B_{\alpha}(H) \equiv\left\{\rho^{\alpha}, H_{0}\right\},
$$

$K=\frac{1}{2}\left(A_{\alpha}(X)+A_{1-\alpha}(X)\right) x+\frac{1}{2}\left(B_{\alpha}(Y)+B_{1-\alpha}(Y)\right)$.

It follows from $K^{*}=K$ that

$$
\begin{aligned}
0 \leq & \operatorname{Tr}\left[K K^{*}\right] \\
= & \frac{1}{4} \operatorname{Tr}\left[\left(A_{\alpha}(X)+A_{1-\alpha}(X)\right)^{2}\right] x^{2} \\
& +\frac{1}{2} \operatorname{Tr}\left[\left(A_{\alpha}(X)+A_{1-\alpha}(X)\right)\left(B_{\alpha}(Y)+B_{1-\alpha}(Y)\right)\right] x \\
& +\frac{1}{4} \operatorname{Tr}\left[\left(B_{\alpha}(Y)+B_{1-\alpha}(Y)\right)^{2}\right] \\
= & \left(\frac{1}{4} \operatorname{Tr}\left[A_{\alpha}(X)^{2}+A_{1-\alpha}(X)^{2}\right]+I_{\rho, \alpha}(X)\right) x^{2} \\
& +\frac{1}{2} \operatorname{Tr}\left[\left(A_{\alpha}(X)+A_{1-\alpha}(X)\right)\left(B_{\alpha}(Y)+B_{1-\alpha}(Y)\right)\right] x \\
& +\left(\frac{1}{4} \operatorname{Tr}\left[B_{\alpha}(Y)^{2}+B_{1-\alpha}(Y)^{2}\right]+J_{\rho, \alpha}(Y)\right) .
\end{aligned}
$$

Then

$$
\begin{aligned}
& \frac{1}{4}\left(\operatorname{Tr}\left[\left(A_{\alpha}(X)+A_{1-\alpha}(X)\right)\left(B_{\alpha}(Y)+B_{1-\alpha}(Y)\right)\right]\right)^{2} \\
\leq & 4\left(\frac{1}{4} \operatorname{Tr}\left[A_{\alpha}(X)^{2}+A_{1-\alpha}(X)^{2}+I_{\rho, \alpha}(X)\right)\right. \\
& \left(\frac{1}{4} \operatorname{Tr}\left[B_{\alpha}(Y)^{2}+B_{1-\alpha}(Y)^{2}\right]+J_{\rho, \alpha}(Y)\right) .
\end{aligned}
$$

Now we have

$$
\begin{aligned}
& \operatorname{Tr}\left[\left(A_{\alpha}(X)+A_{1-\alpha}(X)\right)\left(B_{\alpha}(Y)+B_{1-\alpha}(Y)\right)\right] \\
= & \operatorname{Tr}\left[\left(i\left[\rho^{\alpha}, X_{0}\right]+i\left[\rho^{1-\alpha}, X_{0}\right]\right)\left(\left\{\rho^{\alpha}, Y_{0}^{\}}+\left\{\rho^{1-\alpha}, Y_{0}\right\}\right)\right]\right. \\
= & i \operatorname{Tr}\left[\left(\rho^{\alpha}+\rho^{1-\alpha}\right)^{2} X_{0} Y_{0}-Y_{0} X_{0}\left(\rho^{\alpha}+\rho^{1-\alpha}\right)^{2}\right] \\
= & \operatorname{Tr}\left[\left(\rho^{\alpha}+\rho^{1-\alpha}\right)^{2}\left(i\left[X_{0}, Y_{0}\right]\right)\right] \\
= & \operatorname{Tr}\left[\left(\rho^{\alpha}+\rho^{1-\alpha}\right)^{2}(i[X, Y])\right] .
\end{aligned}
$$

Then (5) is equivalent to the following;

$$
\begin{aligned}
& \frac{1}{4}\left(\operatorname{Tr}\left[\left(\rho^{\alpha}+\rho^{1-\alpha}\right)^{2}(i[X, Y])\right]\right)^{2} \\
\leq & 4\left(\frac{1}{4} \operatorname{Tr}\left[A_{\alpha}(X)^{2}+A_{1-\alpha}(X)^{2}\right]+I_{\rho, \alpha}(X)\right) \\
& \left(\frac{1}{4} \operatorname{Tr}\left[B_{\alpha}(Y)^{2}+B_{1-\alpha}(Y)^{2}\right]+J_{\rho, \alpha}(Y)\right) .
\end{aligned}
$$

And we also have

$$
\begin{aligned}
& \frac{1}{4}\left|\operatorname{Tr}\left[\left(\rho^{\alpha}+\rho^{1-\alpha}\right)^{2}(i[X, Y])\right]\right|^{2} \\
\leq & 4\left(\frac{1}{4} \operatorname{Tr}\left[A_{\alpha}(Y)^{2}+A_{1-\alpha}(Y)^{2}\right]+I_{\rho, \alpha}(Y)\right) \\
& \left(\frac{1}{4} \operatorname{Tr}\left[B_{\alpha}(X)^{2}+B_{1-\alpha}(X)^{2}\right]+J_{\rho, \alpha}(X)\right) .
\end{aligned}
$$

By taking a square root of $(6) \times(7)$, we have

$$
\begin{aligned}
& \left\{\frac{1}{4}\left(\operatorname{Tr}\left[\left(\rho^{\alpha}+\rho^{1-\alpha}\right)^{2}(i[X, Y])\right]\right)^{2}\right\}^{2} \\
\leq \quad & \left(\frac{1}{4} \operatorname{Tr}\left[A_{\alpha}(X)^{2}+A_{1-\alpha}(X)^{2}\right]+I_{\rho, \alpha}(X)\right) \\
& \left(\frac{1}{4} \operatorname{Tr}\left[B_{\alpha}(Y)^{2}+B_{1-\alpha}(Y)^{2}\right]+J_{\rho, \alpha}(Y)\right) \\
& 4\left(\frac{1}{4} \operatorname{Tr}\left[A_{\alpha}(Y)^{2}+A_{1-\alpha}(Y)^{2}\right]+I_{\rho, \alpha}(Y)\right) \\
& \left(\frac{1}{4} \operatorname{Tr}\left[B_{\alpha}(X)^{2}+B_{1-\alpha}(X)^{2}\right]+J_{\rho, \alpha}(X)\right) .
\end{aligned}
$$

Thus

$$
\begin{aligned}
& \frac{1}{4}\left(\operatorname{Tr}\left[\left(\rho^{\alpha}+\rho^{1-\alpha}\right)^{2}(i[X, Y])\right]\right)^{2} \\
\leq & 2 \sqrt{\left(\frac{1}{4} \operatorname{Tr}\left[A_{\alpha}(X)^{2}+A_{1-\alpha}(X)^{2}\right]+I_{\rho, \alpha}(X)\right)} \\
& \sqrt{\left(\frac{1}{4} \operatorname{Tr}\left[B_{\alpha}(Y)^{2}+B_{1-\alpha}(Y)^{2}\right]+J_{\rho, \alpha}(Y)\right)} \\
& \sqrt{\left(\frac{1}{4} \operatorname{Tr}\left[A_{\alpha}(Y)^{2}+A_{1-\alpha}(Y)^{2}\right]+I_{\rho, \alpha}(Y)\right)} \\
& \sqrt{\left(\frac{1}{4} \operatorname{Tr}\left[B_{\alpha}(X)^{2}+B_{1-\alpha}(X)^{2}\right]+J_{\rho, \alpha}(X)\right)} .
\end{aligned}
$$

Therefore

$$
\begin{gathered}
\frac{1}{4}\left(\operatorname{Tr}\left[\left(\frac{\rho^{\alpha}+\rho^{1-\alpha}}{2}\right)^{2}(i[X, Y])\right]\right)^{2} \\
\leq W_{\alpha}(\rho, X) W_{\alpha}(\rho, Y) .
\end{gathered}
$$


Since

$$
\begin{aligned}
& \operatorname{Tr}\left[\left(\frac{\rho^{\alpha}+\rho^{1-\alpha}}{2}\right)^{2}[X, Y]\right] \\
= & -\operatorname{Tr}\left[\left(\frac{\rho^{\alpha}+\rho^{1-\alpha}}{2}\right)^{2}[X, Y]\right],
\end{aligned}
$$

we have

$$
\operatorname{Re}\left[\left(\frac{\rho^{\alpha}+\rho^{1-\alpha}}{2}\right)^{2}[X, Y]\right]=0
$$

And then

$$
\begin{aligned}
& \operatorname{Tr}\left[\left(\frac{\rho^{\alpha}+\rho^{1-\alpha}}{2}\right)^{2}[X, Y]\right] \\
= & i \operatorname{Im} \operatorname{Tr}\left[\left(\frac{\rho^{\alpha}+\rho^{1-\alpha}}{2}\right)^{2}[X, Y]\right] .
\end{aligned}
$$

Hence

$$
\begin{aligned}
& \left(\operatorname{Tr}\left[\left(\frac{\rho^{\alpha}+\rho^{1-\alpha}}{2}\right)^{2}(i[X, Y])\right]\right)^{2} \\
= & -\left(\operatorname{Tr}\left[\left(\frac{\rho^{\alpha}+\rho^{1-\alpha}}{2}\right)^{2}[X, Y]\right]\right)^{2} \\
= & -\left(i \operatorname{Im} \operatorname{Tr}\left[\left(\frac{\rho^{\alpha}+\rho^{1-\alpha}}{2}\right)^{2}[X, Y]\right)^{2}\right. \\
= & \left(\operatorname{Im} \operatorname{Tr}\left[\left(\frac{\rho^{\alpha}+\rho^{1-\alpha}}{2}\right)^{2}[X, Y]\right]\right)^{2} \\
= & \left|\operatorname{Tr}\left[\left(\frac{\rho^{\alpha}+\rho^{1-\alpha}}{2}\right)^{2}[X, Y]\right]\right|^{2} .
\end{aligned}
$$

q.e.d.

We also define the followings to obtain another uncertainty relation.

Definition 2.4 For a quantum state $\rho$ and observable $H$ and $\alpha \in[0,1]$, we define

$$
\begin{aligned}
& \tilde{W}_{\rho, \alpha}(H) \\
\equiv & \frac{1}{4} \sqrt{\operatorname{Tr}\left[\left(i\left[\rho^{\alpha}, H_{0}\right]\right)^{2}\right] \operatorname{Tr}\left[\left(i\left[\rho^{1-\alpha}, H_{0}\right]\right)^{2}\right]} \\
& \sqrt{\operatorname{Tr}\left[\left\{\rho^{\alpha}, H_{0}\right\}^{2}\right] \operatorname{Tr}\left[\left\{\rho^{1-\alpha}, H_{0}\right\}^{2}\right]} .
\end{aligned}
$$

The we have the following theorem.
Theorem 2.5 For a quantum state $\rho$ and observable $X, Y$ and $\alpha \in[0,1]$, we have

$$
\begin{aligned}
& \sqrt{\tilde{W}_{\rho, \alpha}(X) \tilde{W}_{\rho, \alpha}(Y)} \\
\geq & \frac{1}{4}\left|\operatorname{Tr}\left[\rho^{2 \alpha}[X, Y]\right] \operatorname{Tr}\left[\rho^{2(1-\alpha)}[X, Y]\right]\right| .
\end{aligned}
$$

Remark 2.6 There is no relation between Theorem 2.2 and Theorem 2.5 by the following (1), (2).

(1) There is no relation between $4 \tilde{W}_{\rho, \alpha}(X)$ and

$$
\begin{gathered}
\left(\operatorname{Tr}\left[\frac{\left(i\left[\rho^{\alpha}, X_{0}\right]\right)^{2}+\left(i\left[\rho^{1-\alpha}, X_{0}\right]\right)^{2}}{4}\right]+I_{\rho, \alpha}(X)\right) \\
\left(\operatorname{Tr}\left[\frac{\left(\left\{\rho^{\alpha}, X_{0}\right\}\right)^{2}+\left(\left\{\rho^{1-\alpha}, X_{0}\right\}\right)^{2}}{4}\right]+J_{\rho, \alpha}(X)\right) .
\end{gathered}
$$

That is, there are no relation between

$$
\sqrt{\operatorname{Tr}\left[\left(i\left[\rho^{\alpha}, X_{0}\right]\right)^{2}\right] \operatorname{Tr}\left[\left(i\left[\rho^{1-\alpha}, X_{0}\right]\right)^{2}\right]}
$$

and

$$
\begin{aligned}
& \operatorname{Tr}\left[\frac{\left(i\left[\rho^{\alpha}, X_{0}\right]\right)^{2}+\left(i\left[\rho^{1-\alpha}, X_{0}\right]\right)^{2}}{4}\right] \\
& +\frac{1}{2} \operatorname{Tr}\left[\left(i\left[\rho^{\alpha}, X_{0}\right]\right)\left(i\left[\rho^{1-\alpha}, X_{0}\right]\right)\right] .
\end{aligned}
$$

and there is no relation between

$$
\sqrt{\operatorname{Tr}\left[\left\{\rho^{\alpha}, X_{0}\right\}^{2}\right] \operatorname{Tr}\left[\left\{\rho^{1-\alpha}, X_{0}\right\}^{2}\right]}
$$

and

$$
\begin{aligned}
& \operatorname{Tr}\left[\frac{\left\{\rho^{\alpha}, X_{0}\right\}^{2}+\left\{\rho^{1-\alpha}, X_{0}\right\}^{2}}{4}\right] \\
& +\frac{1}{2} \operatorname{Tr}\left[\left\{\rho^{\alpha}, X_{0}\right\}\left\{\rho^{1-\alpha}, X_{0}\right\}\right] .
\end{aligned}
$$

(2) There is no relation between

$$
\left|\operatorname{Tr}\left[\rho^{2 \alpha}[X, Y]\right] \operatorname{Tr}\left[\rho^{2(1-\alpha)}[X, Y]\right]\right|
$$

and

$$
\left|\operatorname{Tr}\left[\left(\frac{\rho^{\alpha}+\rho^{1-\alpha}}{2}\right)^{2}[X, Y]\right]\right|^{2} .
$$

That is, there is no relation between

$$
\left|\operatorname{Tr}\left[\rho^{2 \alpha}[X, Y]\right]\right|
$$

and

$$
\left|\operatorname{Tr}\left[\left(\frac{\rho^{\alpha}+\rho^{1-\alpha}}{2}\right)^{2}[X, Y]\right]\right| .
$$


and there in no relation between

$$
\left|\operatorname{Tr}\left[\rho^{2(1-\alpha)}[X, Y]\right]\right|
$$

and

$$
\left|\operatorname{Tr}\left[\left(\frac{\rho^{\alpha}+\rho^{1-\alpha}}{2}\right)^{2}[X, Y]\right]\right|
$$

(3) When $\alpha=1 / 2$, both Theorem 2.2 and Theorem 2.5 reduce the result of Luo.

Proof of Theorem 2.5. We put

$$
K=i\left[\rho^{\alpha}, X_{0}\right] x+\left\{\rho^{\alpha}, Y_{0}\right\} .
$$

It follows from $K^{*}=K$ that

$$
\begin{aligned}
0 \leq & \operatorname{Tr}\left[K K^{*}\right] \\
= & \operatorname{Tr}\left[\left(i\left[\rho^{\alpha}, X_{0}\right] x+\left\{\rho^{\alpha}, Y_{0}\right\}\right)^{2}\right] \\
= & \operatorname{Tr}\left[\left(i\left[\rho^{\alpha}, X_{0}\right]\right)^{2}\right] x^{2}+2 i \operatorname{Tr}\left[\left[\rho^{\alpha}, X_{0}\right]\left\{\rho^{\alpha}, Y_{0}\right\}\right] x \\
& +\operatorname{Tr}\left[\left\{\rho^{\alpha}, Y_{0}\right\}^{2}\right] \\
= & \operatorname{Tr}\left[\left(i\left[\rho^{\alpha}, X_{0}\right]\right)^{2}\right] x^{2}+2 i i \operatorname{Im} \operatorname{Tr}\left[\rho^{2 \alpha}[X, Y]\right] x \\
& +\operatorname{Tr}\left[\left\{\rho^{\alpha}, Y_{0}\right\}^{2}\right] .
\end{aligned}
$$

Then

$$
\begin{aligned}
& \left|\operatorname{Tr}\left[\rho^{2 \alpha}[X, Y]\right]\right|^{2}=\left(\operatorname{Im} \operatorname{Tr}\left[\rho^{2 \alpha}[X, Y]\right]\right)^{2} \\
& \quad \leq \operatorname{Tr}\left[\left(i\left[\rho^{\alpha}, X_{0}\right]\right)^{2}\right] \operatorname{Tr}\left[\left\{\rho^{\alpha}, Y_{0}\right\}^{2}\right] .
\end{aligned}
$$

By exchanging $X$ and $Y$ we have

$$
\begin{gathered}
\left|\operatorname{Tr}\left[\rho^{2 \alpha}[X, Y]\right]\right|^{2} \\
\leq \operatorname{Tr}\left[\left(i\left[\rho^{\alpha}, Y_{0}\right]\right)^{2}\right] \operatorname{Tr}\left[\left\{\rho^{\alpha}, X_{0}\right\}^{2}\right] .
\end{gathered}
$$

And we also have

$$
\begin{gathered}
\left|\operatorname{Tr}\left[\rho^{2(1-\alpha)}[X, Y]\right]\right|^{2} \\
\leq \operatorname{Tr}\left[\left(i\left[\rho^{1-\alpha}, X_{0}\right]\right)^{2}\right] \operatorname{Tr}\left[\left\{\rho^{1-\alpha}, Y_{0}\right\}^{2}\right] .
\end{gathered}
$$

By exchanging $X$ and $Y$ we have

$$
\begin{gathered}
\left|\operatorname{Tr}\left[\rho^{2(1-\alpha)}[X, Y]\right]\right|^{2} \\
\leq \operatorname{Tr}\left[\left(i\left[\rho^{1-\alpha}, Y_{0}\right]\right)^{2}\right] \operatorname{Tr}\left[\left\{\rho^{1-\alpha}, X_{0}\right\}\right] .
\end{gathered}
$$

We put as follows;

$$
\begin{gathered}
S_{\rho, \alpha}(X) \equiv \frac{1}{2} \operatorname{Tr}\left[\left(i\left[\rho^{\alpha}, X_{0}\right]\right)^{2}\right], \\
S_{\rho, 1-\alpha}(X) \equiv \frac{1}{2} \operatorname{Tr}\left[\left(i\left[\rho^{1-\alpha}, X_{0}\right]\right)^{2}\right],
\end{gathered}
$$

$$
\begin{aligned}
S_{\rho, \alpha}(Y) & \equiv \frac{1}{2} \operatorname{Tr}\left[\left(i\left[\rho^{\alpha}, Y_{0}\right]\right)^{2}\right], \\
S_{\rho, 1-\alpha}(Y) & \equiv \frac{1}{2} \operatorname{Tr}\left[\left(i\left[\rho^{1-\alpha}, Y_{0}\right]\right)^{2}\right], \\
T_{\rho, \alpha}(X) & \equiv \frac{1}{2} \operatorname{Tr}\left[\left\{\rho^{\alpha}, X_{0}\right\}^{2}\right], \\
T_{\rho, 1-\alpha}(X) & \equiv \frac{1}{2} \operatorname{Tr}\left[\left\{\rho^{1-\alpha}, X_{0}\right\}^{2}\right], \\
T_{\rho, \alpha}(Y) & \equiv \frac{1}{2} \operatorname{Tr}\left[\left\{\rho^{\alpha}, Y_{0}\right\}^{2}\right], \\
T_{\rho, 1-\alpha}(Y) & \equiv \frac{1}{2} \operatorname{Tr}\left[\left\{\rho^{1-\alpha}, Y_{0}\right\}^{2}\right] .
\end{aligned}
$$

Then we have

$$
\begin{gathered}
\left|\operatorname{Tr}\left[\rho^{2 \alpha}[X, Y]\right]\right|^{2} \\
\leq 4 \sqrt{S_{\rho, \alpha}(X) T_{\rho, \alpha}(X) S_{\rho, \alpha}(Y) T_{\rho, \alpha}(Y)} . \\
\leq\left.\operatorname{Tr}\left[\rho^{2(1-\alpha)}[X, Y]\right]\right|^{2} \\
\leq 4 \sqrt{S_{\rho, 1-\alpha}(X) T_{\rho, 1-\alpha}(X) S_{\rho, 1-\alpha}(Y) T_{\rho, 1-\alpha}(Y)} .
\end{gathered}
$$

By putting

$$
\begin{gathered}
\tilde{W}_{\rho, \alpha}(X) \equiv \sqrt{S_{\rho, \alpha}(X) S_{\rho, 1-\alpha}(X) T_{\rho, \alpha}(X) T_{\rho, 1-\alpha}(X)}, \\
\tilde{W}_{\rho, \alpha}(Y) \equiv \sqrt{S_{\rho, \alpha}(Y) S_{\rho, 1-\alpha}(Y) T_{\rho, \alpha}(Y) T_{\rho, 1-\alpha}(Y)},
\end{gathered}
$$

we have

$$
\begin{gathered}
\sqrt{\tilde{W}_{\rho, \alpha}(X) \tilde{W}_{\rho, \alpha}(Y)} \\
\geq \frac{1}{4}\left|\operatorname{Tr}\left[\rho^{2 \alpha}[X, Y]\right] \operatorname{Tr}\left[\rho^{2(1-\alpha)}[X, Y]\right]\right| .
\end{gathered}
$$

q.e.d.

\section{A GENERALIZED FISHER INFORMA- TION AND A GENERALIZED CRAMÉR- RAO INEQUALITY}

We review the Fisher information and the CramérRao inequality in quantum mechanical system. We consider the set of all quantum states:

$$
\mathcal{S}_{\theta}(\mathcal{H}) \equiv\left\{\rho_{\theta} \in B(\mathcal{H}) \mid \rho_{\theta} \geq 0, \operatorname{Tr}\left[\rho_{\theta}\right]=1\right\},
$$

with one parameter $\theta \in \mathbb{R}$. Let $H \in \mathcal{L}_{h}(\mathcal{H}) \equiv\{H \in$ $\left.B(\mathcal{H}) \mid H=H^{*}\right\}$ be an estimater of the parameter $\theta$. In the sequel, we consider the case which an estimater is unbiased, that is, $E_{\theta}[H] \equiv \operatorname{Tr}\left[\rho_{\theta} H\right]=\theta$. The variance $V_{\theta}[H]$ of the estimater $H$ is defined by $V_{\theta}[H] \equiv \operatorname{Tr}\left[\rho_{\theta}\left(H-\operatorname{Tr}\left[\rho_{\theta} H\right] I\right)^{2}\right]$. Then the famous Cramér-Rao inequality, which is a relation between the Fisher information and the variance, $V_{\theta}[H] \geq \frac{1}{I\left(\rho_{\theta}, L_{\theta}\right)}$ holds. We should note that the logarithmic derivative 
$L_{\theta} \in B(\mathcal{H})$ is not uniquely determined. Thus we define the symmetric logarithmic derivative $L_{\theta}^{S} \in \mathcal{L}_{h}(\mathcal{H})$ by

$$
\frac{\partial \rho_{\theta}}{\partial \theta} \equiv \frac{1}{2}\left(\rho_{\theta} L_{\theta}^{S}+L_{\theta}^{S} \rho_{\theta}\right)
$$

Then the symmetric logarithmic derivative $L_{\theta}^{S}$ is uniquely determined $[1,4,5]$ and we have

$$
I\left(\rho_{\theta}, L_{\theta}\right) \geq I\left(\rho_{\theta}, L_{\theta}^{S}\right)
$$

In addition, for the symmetric logarithmic derivative $L_{\theta}^{S}$, we have the Cramér-Rao inequality $[1,4,5]$ :

$$
V_{\theta}[H] \geq \frac{1}{I\left(\rho_{\theta}, L_{\theta}^{S}\right)} .
$$

Due to the inequality (8), we have the following theorem known as Cramér-Rao inequality.

\section{Theorem 3.1}

$$
V_{\theta}[H] \geq \frac{1}{I\left(\rho_{\theta}, L_{\theta}\right)}
$$

That is, the symmetric logarithmic derivative $L_{\theta}^{S}$ gives the best estimation of the lower bound for the variance $V_{\theta}[H]$.

We here introduce a generalized Fisher information with one-parameter $\alpha \in[0,1]$.

Definition 3.2 We define a generalized Fisher information by

$$
I_{\alpha}\left(\rho_{\theta}, L_{\theta, \alpha}\right) \equiv \operatorname{Tr}\left[\rho_{\theta}^{\alpha} L_{\theta, \alpha} \rho_{\theta}^{1-\alpha} L_{\theta, \alpha}^{*}\right], \alpha \in[0,1],
$$

where a generalized logarithmic derivative $L_{\theta, \alpha}$ is defined by

$$
\frac{\partial \rho_{\theta}}{\partial \theta} \equiv \frac{1}{2}\left(\rho_{\theta}^{\frac{1+\alpha}{2}} L_{\theta, \alpha} \rho_{\theta}^{\frac{1-\alpha}{2}}+\rho_{\theta}^{\frac{1-\alpha}{2}} L_{\theta, \alpha}^{*} \rho_{\theta}^{\frac{1+\alpha}{2}}\right) .
$$

Note that $\alpha=1$ or $\left[\rho_{\theta}, L_{\theta, \alpha}\right]=0$ recovers $I_{\alpha}\left(\rho_{\theta}, L_{\theta, \alpha}\right)=I\left(\rho_{\theta}, L_{\theta}\right)$. We also have $I_{\alpha}\left(\rho_{\theta}, L_{\theta, \alpha}\right) \geq 0$ and the following trace inequality.

Theorem 3.3 For a self-adjoint operator $H$, a density operator $\rho_{\theta}$ with the parameter $\theta$ and $\alpha \in[0,1]$, if we have $E_{\theta}[H]=\theta$, then we have the inequality

$$
V_{\theta}[H] \geq \frac{1}{I_{\alpha}\left(\rho_{\theta}, L_{\theta, \alpha}\right)} .
$$

It is clear that (11) is obtained by putting

$$
L_{\rho, \theta}=\rho_{\theta}^{\frac{1-\alpha}{2}} L_{\theta} \rho_{\theta}^{\frac{\alpha-1}{2}} .
$$

\section{References}

[1] S.Amari and H.Nagaoka, Methods of information geometry, AMS Oxford University Press, 2000.

[2] P.Gibilisco, D.Imparato and T.Isola, A Robertson-type uncertainty principle and quantum Fisher information, Linear Alg. Appl., vol.428(2008), pp.1706-1724.

[3] F.Hansen, Metric adjusted skew information, arXiv:math-ph/0607049v4.

[4] C.W.Helstrom, Quantum detection and estimation theory, Academic Press, 1976.

[5] A.S.Holevo, Probabilistic and statistical aspects of quantum theory, North-Holland, 1982.

[6] H.Kosaki, Matrix trace inequality related to uncertainty principle, International J. Math., vol.16(2005), pp.629-646.

[7] E.H.Lieb, Convex trace functions and the Wigner-Yanase-Dyson conjecture, Adv. Math., vol.11(1973), pp.267-288.

[8] S.Luo, Heisenberg uncertainty relation for mixed states, Phys. Rev.A, vol.72(2005), 042110.

[9] S.Luo and Q.Zhang, On skew information, IEEE Trans. Information Theory, vol.50(2004), pp.1778-1782, and Correction to "On skew information", IEEE Trans. Information Theory, vol.51(2005), p.4432.

[10] E.P.Wigner and M.M.Yanase, Information content of distribution, Proc. Nat. Acad. Sci, U.S.A., vol.49(1963), pp.910-918.

[11] K.Yanagi, S.Furuichi and K.Kuriyama, A generalization skew information and uncertainty relation, IEEE Trans. Information Theory, vol.51(2005), pp.4401-4404. 